\title{
SHARP BOUNDS FOR THE FIRST ZAGREB INDEX AND FIRST ZAGREB COINDEX
}

\author{
EMINA I. MILOVANOVIĆ AND IGOR Ž. MILOVANOVIĆ
}

Received 07 June, 2014

\begin{abstract}
Let $G$ be an undirected connected graph with $n, n \geq 2$, vertices and $m$ edges with vertex degrees $\Delta=d_{1} \geq d_{2} \geq \cdots \geq d_{n}=\delta$. Lower and upper bounds of a graph invariants $M_{1}=\sum_{i=1}^{n} d_{i}^{2}$, referred to as the first Zagreb index, and $\bar{M}_{1}=\sum_{i \nsim j}\left(d_{i}+d_{j}\right)$, named the first Zagreb coindex, depending on parameters $n, m, \Delta$ and $\delta$ are obtained. The obtained results represent improvement of the results reported in the literature.
\end{abstract}

2010 Mathematics Subject Classification: 05C35

Keywords: first Zagreb index, first Zagreb coindex, degree (of vertex)

\section{INTRODUCTION}

Let $G$ be an undirected connected graph with $n, n \geq 2$, vertices and $m$ edges, and let $\Delta=d_{1} \geq d_{2} \geq \ldots \geq d_{n}=\delta>0$ be the sequence of its vertex degrees.

In graph theory, a graph invariant is a property of graphs that does not depend on graph representations, such as particular labeling of its vertices or drawings of the graph. A number of different invariants have been introduced so far. One of the oldest was introduced by Gutman and Trinajstić in 1972, known under the name first Zagreb index, defined as the sum of squares of vertex degrees of graph, i.e. as

$$
M_{1}=\sum_{i=1}^{n} d_{i}^{2}
$$

In [8] Došlić proposed a new graph invariant named first Zagreb coindex, defined as

$$
\bar{M}_{1}=\sum_{i \nsim j}\left(d_{i}+d_{j}\right) .
$$

These graph invariants play an important role in many scientific areas, notably in chemistry and network theory (see for example [12] and [1,18]). In earlier works [2, 3, 5-7, 9-17, 19], several bounds for $M_{1}$ and $\bar{M}_{1}$ were reported. These depend on usual structural parameters (number of vertices, number of edges, vertex degrees, and similar). 
In this paper we consider the problem of finding lower and upper bounds of a graph invariants $M_{1}$ and $\bar{M}_{1}$. The obtained results represent improvement of the results reported in the literature.

\section{PRELIMinaries}

Here we recall some results from spectral graph theory, and state a few analytical inequalities needed for our work.

Lemma 1 ([9, 16]). Let $G$ be an undirected connected graph with $n, n \geq 2$, vertices and $m$ edges. Then

$$
M_{1} \geq \frac{4 m^{2}}{n}
$$

Equality holds if and only if $G$ is isomorph with a regular graph.

Lemma 2 ([10]). Let $G$ be simple graph with $n$, vertices and $m$ edges. Then

$$
M_{1} \leq \frac{4 m^{2}}{n}+\frac{n}{4}(\Delta-\delta)^{2}
$$

Lemma 3 ([10,11,15,17]). Let $G$ be simple graph with $n$ vertices and $m$ edges. Then

$$
M_{1} \leq \frac{m^{2}}{n}\left(\sqrt{\frac{\Delta}{\delta}}+\sqrt{\frac{\delta}{\Delta}}\right)^{2}
$$

with equality if and only if $G$ is regular graph, or $G$ is bidegreed graph such that $\Delta+\delta$ divides $\delta n$ and there are exactly $p=\frac{\delta n}{\Delta+\delta}$ vertices of degree $\Delta$, and $q=\frac{\Delta n}{\Delta+\delta}$ vertices of degree $\delta$.

Note that complete conditions when equality in (2.3) occurs were given only in [15].

Lemma 4 ([2]). Suppose $G$ is a connected graph with exactly $n$ vertices and $m$ edges. Then we have

$$
\bar{M}_{1}=2 m(n-1)-M_{1} .
$$

Lemma 5 ([14]). Let $G$ be a simple graph with $n$ vertices and $m$ edges. Then

$$
\bar{M}_{1} \leq-\frac{4 m^{2}}{n}+2 m(n-1),
$$

and

$$
\bar{M}_{1} \geq 2 m(n-1)-\frac{(\Delta+\delta)^{2}}{n \Delta \delta} m^{2} .
$$

The equality holds if and only if $G$ is regular. 
Lemma 6 ([4]). Let $p_{1}, p_{2}, \ldots, p_{n}$ be non-negative real numbers and $a_{1}, a_{2}, \ldots, a_{n}$ and $b_{1}, b_{2}, \ldots, b_{n}$, real numbers with the properties

$$
0<r_{1} \leq a_{i} \leq R_{1}<+\infty \quad \text { and } \quad 0<r_{2} \leq b_{i} \leq R_{2}<+\infty
$$

for each $i=1,2, \ldots, n$. Further, let $S$ be a subset of $I_{n}=\{1,2, \ldots, n\}$ which minimizes the expression

$$
\left|\sum_{i \in S} p_{i}-\frac{1}{2} \sum_{i=1}^{n} p_{i}\right| \text {. }
$$

Then

$$
\left|\sum_{i=1}^{n} p_{i} \sum_{i=1}^{n} p_{i} a_{i} b_{i}-\sum_{i=1}^{n} p_{i} a_{i} \sum_{i=1}^{n} p_{i} b_{i}\right| \leq\left(R_{1}-r_{1}\right)\left(R_{2}-r_{2}\right) \sum_{i \in S} p_{i}\left(\sum_{i=1}^{n} p_{i}-\sum_{i \in S} p_{i}\right) \text {. }
$$

\section{MAIN RESUlts}

\subsection{The first Zagreb index}

The following theorem establishes bound for $M_{1}$ depending on parameters $n, m$, $\Delta$ and $\delta$.

Theorem 1. Let $G$ be an undirected connected graph with $n, n \geq 2$, vertices and $m$ edges. Then

$$
M_{1} \geq \frac{4 m^{2}}{n}+\frac{1}{2}(\Delta-\delta)^{2} .
$$

Equality holds if and only if $G$ is isomorph with $k$-regular graph, $1 \leq k \leq n-1$.

Proof. From equality

$$
n M_{1}-4 m^{2}=n \sum_{i=1}^{n} d_{i}^{2}-\left(\sum_{i=1} d_{i}\right)^{2}=\sum_{1 \leq i \leq j \leq n}\left(d_{i}-d_{j}\right)^{2}
$$

and inequality

$$
\begin{aligned}
\sum_{1 \leq i<j \leq n}\left(d_{i}-d_{j}\right)^{2} & \geq \sum_{i=2}^{n-1}\left(\left(d_{1}-d_{i}\right)^{2}+\left(d_{i}-d_{n}\right)^{2}\right)+\left(d_{1}-d_{n}\right)^{2} \\
& \geq \sum_{i=2}^{n-1} \frac{1}{2}\left(d_{1}-d_{n}\right)^{2}+\left(d_{1}-d_{n}\right)^{2} \\
& =\frac{n-2}{2}(\Delta-\delta)^{2}+(\Delta-\delta)^{2}=\frac{n}{2}(\Delta-\delta)^{2}
\end{aligned}
$$

the inequality (3.1) is obtained.

Since equality in (3.2) holds if and only if $d_{1}=d_{2}=\cdots=d_{n}$, equality in (3.1) holds if and only if $G$ is isomorph with $k$-regular graph, $1 \leq k \leq n-1$. 
Remark 1. Since $(\Delta-\delta)^{2} \geq 0$, the inequality (3.1) is stronger than inequality (2.1).

Theorem 2. Let $G$ be an undirected connected graph with $n, n \geq 2$, vertices and $m$ edges. Then

where

$$
M_{1} \leq \frac{4 m^{2}}{n}+n(\Delta-\delta)^{2} \alpha(n)
$$

$$
\alpha(n)=\frac{1}{4}\left(1-\frac{1+(-1)^{n+1}}{2 n^{2}}\right) .
$$

Equality in (3.3) holds if and only if $G$ is isomorph with $k$-regular graph, $1 \leq k \leq$ $n-1$.

Proof. Suppose that $S$ is a subset of the form $S=\{1,2, \ldots, k\} \subset I_{n}=\{1,2, \ldots, n\}$ and that $p_{i}=1, i=1,2, \ldots, n$. Then the expression (2.7) reaches the minimum if $k=\left\lfloor\frac{n}{2}\right\rfloor$, i.e. if $S=\left\{1,2, \ldots,\left\lfloor\frac{n}{2}\right\rfloor\right\}$. Now, for $p_{i}=1, a_{i}=d_{i}, b_{i}=d_{i}, i=1,2, \ldots, n$, $r_{1}=r_{2}=\delta, R_{1}=R_{2}=\Delta$ and $S=\left\{1,2, \ldots,\left\lfloor\frac{n}{2}\right\rfloor\right\}$, the equality (2.8) transforms into

$$
n \sum_{i=1}^{n} d_{i}^{2}-\left(\sum_{i=1}^{n} d_{i}\right)^{2} \leq(\Delta-\delta)^{2}\left\lfloor\frac{n}{2}\right\rfloor\left(n-\left\lfloor\frac{n}{2}\right\rfloor\right),
$$

i.e.

Since

$$
n M_{1}-4 m^{2} \leq n^{2}(\Delta-\delta)^{2} \frac{1}{n}\left\lfloor\frac{n}{2}\right\rfloor\left(1-\frac{1}{n}\left\lfloor\frac{n}{2}\right\rfloor\right) .
$$

$$
\alpha(n)=\frac{1}{n}\left\lfloor\frac{n}{2}\right\rfloor\left(1-\frac{1}{n}\left\lfloor\frac{n}{2}\right\rfloor\right)=\frac{1}{4}\left(1-\frac{1+(-1)^{n+1}}{2 n^{2}}\right)
$$

from the above inequality we obtain (3.3).

Since equality in (3.4) holds if and only if $d_{1}=d_{2}=\cdots=d_{n}$, equality in (3.3) holds if and only if $G$ is isomorph with $k$-regular graph, $1 \leq k \leq n-1$.

Remark 2. If $n$ is even number, $n \geq 2$, then $\alpha(n)=\frac{1}{4}$, and if $n$ is odd, $n \geq 3$, then $\alpha(n)=\frac{(n-1)(n+1)}{4 n^{2}}<\frac{1}{4}$. This means that inequality (3.3) is stronger than inequality (2.2) for each odd $n, n \geq 3$.

Theorem 3. Let $G$ be an undirected connected graph with $n, n \geq 2$, vertices and $m$ edges. Further, let $S$ be a subset of $I_{n}=\{1,2, \ldots, n\}$ that minimizes the expression

$$
\left|\sum_{i \in S} d_{i}-m\right| \text {. }
$$

Then

$$
M_{1} \leq \frac{4 m^{2}\left(1+\left(\sqrt{\frac{\Delta}{\delta}}-\sqrt{\frac{\delta}{\Delta}}\right)^{2} \beta(S)\right)}{n}
$$


where

$$
\beta(S)=\frac{1}{2 m} \sum_{i \in S} d_{i}\left(1-\frac{1}{2 m} \sum_{i \in S} d_{i}\right) .
$$

Equality in (3.6) holds if and only if $G$ is isomorph with $k$-regular graph, $1 \leq k \leq$ $n-1$, or bidegreed graph such that $\Delta+\delta$ divides $n \delta$ and there are exactly $p=\frac{n \delta}{\Delta+\delta}$ vertices of degree $\Delta$ and $q=\frac{n \Delta}{\Delta+\delta}$ vertices of degree $\delta$.

Proof. For $p_{i}=d_{i}, i=1,2, \ldots, n$, the expression (2.7) transforms into (3.5). Suppose that $S$ is subset of $I_{n}=\{1,2, \ldots, n\}$ for which the expression (3.5) reaches a minimum. Now for $p_{i}=d_{i}, a_{i}=d_{i}, b_{i}=\frac{1}{d_{i}}, i=1,2, \ldots, n, r_{1}=\delta, R_{1}=\Delta$, $r_{2}=\frac{1}{\Delta}$ and $R_{2}=\frac{1}{\delta}$ the inequality (2.8) becomes

$$
n \sum_{i=1}^{n} d_{i}^{2}-\left(\sum_{i=1}^{n} d_{i}\right)^{2} \leq(\Delta-\delta)\left(\frac{1}{\delta}-\frac{1}{\Delta}\right) \sum_{i \in S} d_{i}\left(2 m-\sum_{i \in S} d_{i}\right)
$$

i.e.

$$
n M_{1}-4 m^{2} \leq 4 m^{2}\left(\sqrt{\frac{\Delta}{\delta}}-\sqrt{\frac{\delta}{\Delta}}\right)^{2} \beta(S)
$$

wherefrom the inequality (3.6) is obtained.

Equality in (3.7) holds if and only if $d_{1}=d_{2}=\cdots=d_{n}$, therefore the equality in (3.6) holds if and only if $G$ is isomorph with $k$-regular graph, $1 \leq k \leq n-1$.

Suppose $\Delta+\delta$ divides $n \delta$ and that graph $G_{1}$ has exactly $p=\frac{n \delta}{n+\delta}$ vertices of degree $\Delta$ and $q=\frac{n \Delta}{\Delta+\delta}$ vertices of degree $\delta$, where $p+q=n$. In that case $m=\frac{n \delta \Delta}{\Delta+\delta}$ and expression (3.5) reaches the minimum if $S=\{1,2, \ldots, p\}$ and $\beta(s)=\frac{1}{4}$. This means that equality in (3.5) holds if and only if $G$ is isomorph with bidegreed graph $G_{1}$.

Remark 3. Since for each set $S \subset I_{n}=\{1,2, \ldots, n\}$ holds $\beta(S) \leq \frac{1}{4}$, we have that

$$
\frac{4 m^{2}\left(1+\left(\sqrt{\frac{\Delta}{\delta}}-\sqrt{\frac{\delta}{\Delta}}\right)^{2} \beta(S)\right)}{n} \leq \frac{m^{2}\left(\sqrt{\frac{\Delta}{\delta}}+\sqrt{\frac{\delta}{\Delta}}\right)^{2}}{n} .
$$

This means that inequality (3.6) is stronger than (2.3).

Corollary 1. Let $G$ be an undirected connected graph with $n, n \geq 2$, vertices and $m$ edges. If $\delta=1$ then

$$
M_{1} \leq \frac{4 m^{2}\left(1+\frac{(n-2)^{2}}{n-1} \beta(S)\right)}{n}
$$


Equality holds if and only if $G$ is isomorph with $K_{1, n-1}$. If $\delta \geq 2$, then

$$
M_{1} \leq \frac{4 m^{2}\left(1+\frac{(n-3)^{2}}{2(n-1)} \beta(S)\right)}{n} .
$$

Equality holds if and only if $G$ is isomorph with graph $K_{3}$.

Remark 4. Since $\beta(S) \leq \frac{1}{4}$, it follows that inequalities (3.8) and (3.9) are stronger of the corresponding proved in [15] (their Corollary 2.1) and [17] (their Corollary 2.3).

\subsection{First Zagreb coindex}

Now we give a theorems which provides a lower and upper bounds for $\bar{M}_{1}$ in terms of parameters $n, m, \Delta$ and $\delta$.

Theorem 4. Let $G$ be an undirected connected graph with $n, n \geq 2$, vertices and $m$ edges. Then

$$
\bar{M}_{1} \leq \frac{2 m}{n}(n(n-1)-2 m)-\frac{1}{2}(\Delta-\delta)^{2} .
$$

Equality holds if and only if $G$ is isomorph with $k$-regular graph, $1 \leq k \leq n-1$.

Proof. The proof immediately follows by Lemma 4 and Theorem 1.

Remark 5. The inequality (3.10) is stronger than (2.5).

Theorem 5. Let $G$ be an undirected connected graph with $n, n \geq 2$, vertices and $m$ edges. Then

$$
\bar{M}_{1} \geq \frac{2 m}{n}(n(n-1)-2 m)-n(\Delta-\delta)^{2} \alpha(n) .
$$

Equality holds if and only if $G$ is isomorph with $k$-regular graph, $1 \leq k \leq n-1$.

Proof. The proof immediately follows by Lemma 4 and Theorem 2.

Theorem 6. Let $G$ be an undirected connected graph with $n, n \geq 2$, vertices and $m$ edges. Further, let $S$ be a subset of $I_{n}=\{1,2, \ldots, n\}$ that minimizes the expression

$$
\left|\sum_{i \in S} d_{i}-m\right|
$$

Then

$$
\bar{M}_{1} \geq 2 m(n-1)-\frac{4 m^{2}\left(1+\left(\sqrt{\frac{\Delta}{\delta}}-\sqrt{\frac{\delta}{\Delta}}\right)^{2} \beta(S)\right)}{n}
$$

where $\beta(s)=\frac{1}{2 m} \sum_{i \in S} d_{i}\left(1-\frac{1}{2 m} \sum_{i \in S} d_{i}\right)$. 
Equality in (3.11) holds if and only if $G$ is isomorph with $k$-regular graph, $1 \leq k \leq$ $n-1$, or bidegreed graph such that $\Delta+\delta$ divides $n \delta$ and there are exactly $p=\frac{n \bar{\delta}}{\delta+\delta}$ vertices of degree $\Delta$ and $q=\frac{n \Delta}{\Delta+\delta}$ vertices of degree $\delta$.

Proof. The proof immediately follows by Lemma 4 and Theorem 3.

Remark 6. Inequality (3.11) is stronger than (2.6).

\section{ACKNOWLEDGEMENTS}

This work was supported by the Serbian Ministry of Education and Science, Project No TR32012 and TR32009.

\section{REFERENCES}

[1] A. Arenas, L. Danon, A. Diaz-Guilera, P. M. Gleiser, and R. Guimera, "Community analysis in social networks," European Physical J. B., vol. 38, no. 3, pp. 373-380, 2004, doi: 10.1140/epjb/e2004-00130-1.

[2] A. R. Ashrafi, T. Doslić, and H. A., "The zagreb coindices of graph operations," Discrete Appl. Math., vol. 158, no. 15, pp. 1571-1580, 2010, doi: 10.1016/j.dam.2010.05.017.

[3] D. D. Caen, "An upper bound on the sum of squares of degrees in a graph," Discrete Math., vol. 185, no. 1-3, pp. 245-248, 1998, doi: 10.1016/S0012-365X(97)00213-6.

[4] P. Cerone and S. S. Dragomir, "A refinement of the grüss inequality and applications," J. Ineq. Pure Appl. Math, vol. 5, no. 3, pp. 245-248, 2002.

[5] S. M. Cioaba, "Sums of powers of the degrees of graph," Discrete Math., vol. 306, no. 16, pp. 1959-1964, 2006, doi: 10.1016/j.disc.2006.03.054.

[6] K. C. Das, "Sharp bound for the sum of the squares of the degrees of a graph," Kragujevac J. Math., vol. 25, pp. 31-49, 2003.

[7] K. C. Das, "Maximizing the sum of squares of graph," Discrete Math., vol. 285, no. 1-3, pp. 57-66, 2004, doi: 10.1016/j.disc.2004.04.007.

[8] T. Doslić, "Vertex-weighted wiener polynomials for composite graphs," Ars Math. Contemp., vol. 1, no. 1, pp. 66-80, 2008.

[9] C. S. Edwards, "The largest vertex degree sum for a triangle in a graph," Bul. London Math. Soc., vol. 9, pp. 203-208, 1977, doi: 10.1112/blms/9.2.203.

[10] G. H. Fath-Tabar, "Old and new zagreb indices of graphs," MATCH Commun. Math. Comput. Chem., vol. 64, no. 1, pp. 79-84, 2011.

[11] T. Guixian, H. Tingzhy, and C. Shuyu, "Bounds on the algebraic connectivity of graphs," Advanc. Math., vol. 41, no. 2, pp. 217-224, 2012.

[12] I. Gutman and K. C. Das, "The first zagreb index 30 years after," MATCH Commun. Math. Comput. Chem., no. 50, pp. 83-92, 2004.

[13] I. Gutman and N. Trinajstić, "Graph theory and molecular orbitas. total $\pi$-electron energy of alternant hydrocarbons," Chem. Phys. Letters, no. 17, pp. 535-538, 1997, doi: 10.1016/00092614(72)85099-1.

[14] S. Hossein Zadeh, A. Hamzeh, and A. R. Ashrafi, "Extremal properties of zagreb coindices and degree distance of graphs," Miskolc Math. Notes, vol. 11, no. 2, pp. 129-137, 2010.

[15] A. Ilić, M. Ilić, and B. Liu, "On the upper bounds for the first zagreb index," Kragujevac J. Math., vol. 32, no. 1, pp. 173-182, 2011.

[16] A. Ilić and D. Stevanović, "On comparing zagreb indices," MATCH Commun. Math. Comput. Chem., vol. 62, no. 3, pp. 681-687, 2009. 
[17] M. Liu and B. Liu, "New sharp upper bounds for the first zagreb index," MATCH Commun. Math. Comput. Chem., vol. 62, no. 3, pp. 689-698, 2009.

[18] T. M. Truta, A. Campan, and A. L. Ralescu, "Preservation of structural properties in anonymized social networks," in Proc.: IEEE International Conference on Collaborative Computing: Networking, Applications and Worksharing," doi: 10.4108/icst.collaboratecom.2012.250414, Pittsburg, PA, 2012, pp. 619-627.

[19] B. Zhou, "Zagreb indices," MATCH Commun. Math. Comput. Chem., no. 52, pp. 113-118, 2004.

\section{Authors' addresses}

Emina I. Milovanović

University of Niš, Faculty of Electronics Engineering, A. Medvedeva 14, P.O. Box 73, 18000 Niš, Serbia

E-mail address: emadelfak.ni.ac.rs

Igor Ž. Milovanović

University of Niš, Faculty of Electronic Engineering, A. Medvedeva 14, P.O. Box 73, 18000 Niš, Serbia

E-mail address: igoreelfak.ni.ac.rs 